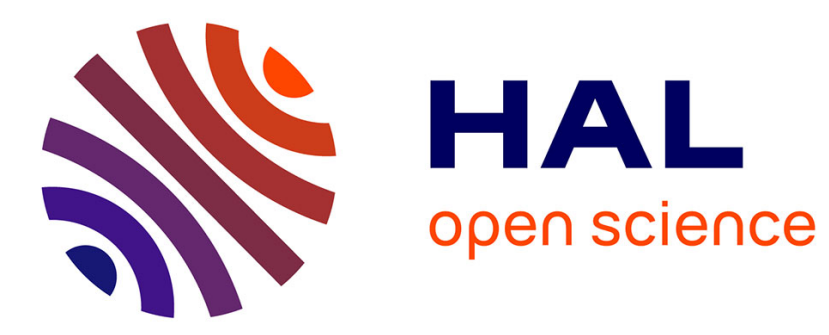

\title{
Effort Dynamics in Supervised Work Groups
}

Arianna Dal Forno, Ugo Merlone

\section{To cite this version:}

Arianna Dal Forno, Ugo Merlone. Effort Dynamics in Supervised Work Groups. Journal of Economic Behavior and Organization, 2010, 75 (3), pp.413. 10.1016/j.jebo.2010.05.010 . hal-00849409

\section{HAL Id: hal-00849409 https://hal.science/hal-00849409}

Submitted on 31 Jul 2013

HAL is a multi-disciplinary open access archive for the deposit and dissemination of scientific research documents, whether they are published or not. The documents may come from teaching and research institutions in France or abroad, or from public or private research centers.
L'archive ouverte pluridisciplinaire HAL, est destinée au dépôt et à la diffusion de documents scientifiques de niveau recherche, publiés ou non, émanant des établissements d'enseignement et de recherche français ou étrangers, des laboratoires publics ou privés. 


\section{Accepted Manuscript}

Title: Effort Dynamics in Supervised Work Groups

Authors: Arianna Dal Forno, Ugo Merlone

PII: $\quad$ S0167-2681(10)00092-2

DOI: $\quad$ doi:10.1016/j.jebo.2010.05.010

Reference: $\quad$ JEBO 2545

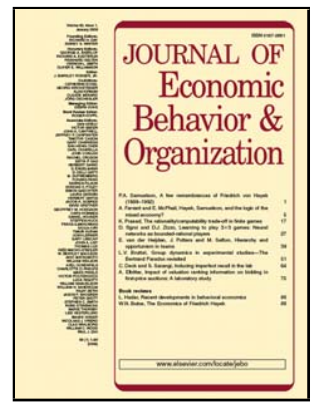

To appear in: Journal of Economic Behavior \& Organization

Received date: $\quad$ 18-3-2009

Revised date: $\quad 10-5-2010$

Accepted date: $\quad 11-5-2010$

Please cite this article as: Dal Forno, A., Merlone, U., Effort Dynamics in Supervised Work Groups, Journal of Economic Behavior and Organization (2008), doi:10.1016/j.jebo.2010.05.010

This is a PDF file of an unedited manuscript that has been accepted for publication. As a service to our customers we are providing this early version of the manuscript. The manuscript will undergo copyediting, typesetting, and review of the resulting proof before it is published in its final form. Please note that during the production process errors may be discovered which could affect the content, and all legal disclaimers that apply to the journal pertain. 


\title{
Effort Dynamics in Supervised Work Groups
}

\author{
Arianna Dal Forno ${ }^{\mathrm{a}, *}$, Ugo Merlone ${ }^{\mathrm{b}}$ \\ a Department of Statistics and Applied Mathematics, University of Torino, Corso \\ Unione Sovietica 218 bis, Torino I-10134, Italy, phone +39011 6705753, fax +39 \\ 0116705783 \\ ${ }^{\mathrm{b}}$ Department of Statistics and Applied Mathematics, University of Torino, Corso \\ Unione Sovietica 218 bis, Torino I-10134, Italy, phone +39011 6705753, fax +39 \\ 0116705783
}

\begin{abstract}
A dynamic model of supervised work group, where the total production is the result of two non-additive tasks, is provided. Taking into account the perception of inequity, as in Adams (1965), we analyze the effort allocation dynamics to resolve the tension in a situation resembling the proverbial "straw that broke the camel's back". The results prove the coexistence of different dynamics varying in terms of efficiency. For some dynamics we can observe retaliation between subordinates and even full conflict with no production.
\end{abstract}

Key words: Inequity, Work Group, Conflict, Dynamical System, Multistability, Organizational Behavior.

\section{Introduction}

In the literature, perceptions of inequity have been associated with substantive employee responses in the workplace. According to Cropanzano and Schminke (1995), in modern justice research the most widely studied allocation rule is that of equity. According to the equity principle, one's outcome should be proportional to one's inputs, that is, those who contribute more to a common task

* Corresponding author.

Email address: dalforno@econ.unito.it (Arianna Dal Forno).

1 The authors are grateful to Laura Gardini and Gian Italo Bischi for helpful suggestions. Also MDEF2008 workshop participants at Urbino University have provided useful comments. 
should reap most of the benefits (Cropanzano and Schminke, 1995). While inequity theory (Adams, 1965) is only one among the different theories, several authors acknowledge its importance in terms of explicitness (Campbell and Pritchard, 1976) and rigour (Mowday, 1979). Finally, its formulation allows the operationalizing of the concept since it is already formalized in terms of a mathematical inequity (see Adams, 1965, pages 280-281). While both the original contribution by Adams and the most commonly used formulations of equity theory rely on a static view, Cosier and Dalton (1983) proposed an interesting reformulation of this theory which considers also the role of time. The model they proposed provides a time dimension by explicitly considering past inequity in order to predict the strength of motivation to reduce it. Among the examples, they gave some situations resembling the pattern of the proverbial "straw that broke the camel's back". These kind of situations can be modeled as a discontinuous function, i.e., an individual who thus far has been tolerant to inequity, suddenly exhibits a rather intolerant behavior.

In this paper we provide a model of a supervised work group where subordinates perform a task with the supervisor and a task with the partner, which both determine the total production. The interaction scheme we consider was studied both empirically and theoretically; among the results, Dal Forno and Merlone (2009b) observe that, with the optimal incentive scheme they derive, subordinates are likely to perceive inequity. In this paper, we extend the static view of the previous research, and analyze the effort dynamics when at least one of the subordinates has exceeded his/her inequity threshold. The structure of the paper is the following. In Section 2 we describe the model of work group we consider and discuss the optimal incentive scheme when subordinates do not respond to inequity. Section 3 discusses how subordinates, responding to inequity, react when they perceive that their colleague allocates effort differently from what they expect. Section 4 examines what the effort allocation dynamics is when different subordinates are matched. Finally, in the last section we discuss our results and examine future lines of research.

\section{The model}

Following Dal Forno and Merlone (2007, 2009a), we consider a model of supervised work group in which a supervisor (acting as the principal) and two subordinates (acting as agents) cooperate. Agent $i$ allocates his effort $l_{i}$ with the partner, and the effort $u_{i}$ with the supervisor. The joint production function for agents 1 and 2 is $\Gamma\left(u_{1}+u_{2}\right)^{\alpha}\left(l_{1}+l_{2}\right)^{\beta}$, where $\Gamma \in \mathbb{R}_{++}$is a constant factor $^{2}$, and $\alpha, \beta \in(0,1)$ are, respectively, the output elasticity with respect to the joint effort with the supervisor and with the partner. As a consequence,

$\overline{2}$ We recall that $\mathbb{R}_{++}$is the set of positive real numbers; the case $\Gamma=0$ is trivial. 
the agents have to decide both how much effort to exert, and how to partition it in the two complementary tasks ${ }^{3}$. Agents bear a cost for effort: agent $i$ 's cost function $c_{i}: \mathbb{R}_{+}^{2} \rightarrow \mathbb{R}_{+}$will be denoted with $c_{i}\left(u_{i}, l_{i}\right)$; cost functions are private information. Furthermore, each agent $i$ can observe the level effort $l_{-i}$ his partner provides with him ${ }^{4}$, but not the one which is provided with the supervisor. Conversely, the supervisor can only observe the joint output and the effort each agent provides with her. The supervisor's profit is a share $\gamma \in(0,1)$ of the supervised work group production minus the incentives she pays to her subordinates. In the following, we assume that the output is sold on market at unitary price and the production and sharing constants $\Gamma$ and $\gamma$ are such that $\Gamma \gamma=1$; this is not restrictive, it simplifies the notation, and allows us to simply consider monetary payoffs. Finally, agents' retribution consists of a fixed wage $w>0$ plus a performance-contingent reward; we assume that the fixed wage is sufficient to meet basic needs, in terms of the hierarchy of needs theory (Maslow, 1970), physiological needs and needs of safety; in economic terms we say that the participation constraint is met. The performance-contingent reward is a linear incentive $b_{g}$ proportional to the joint output of the team and a linear incentive $b_{i}$ on the effort each agent exerts with the supervisor. Therefore, the problem can be formalized as a bilevel programming problem:

$$
\max _{b_{g}, b_{1}, b_{2}}\left(1-2 b_{g}\right)\left(u_{1}+u_{2}\right)^{\alpha}\left(l_{1}+l_{2}\right)^{\beta}-b_{1} u_{1}-b_{2} u_{2}
$$

such that, given $b_{g}, b_{1}, b_{2}$ subordinates solve

$$
\begin{aligned}
& \max _{u_{1}, l_{1}} w+b_{g}\left(u_{1}+u_{2}\right)^{\alpha}\left(l_{1}+l_{2}\right)^{\beta}+b_{1} u_{1}-c_{1}\left(u_{1}, l_{1}\right) \\
& \max _{u_{2}, l_{2}} w+b_{g}\left(u_{1}+u_{2}\right)^{\alpha}\left(l_{1}+l_{2}\right)^{\beta}+b_{2} u_{2}-c_{2}\left(u_{2}, l_{2}\right)
\end{aligned}
$$

For the sake of simplicity we assume $w=0$, this is not restrictive. Examining the form of the problem, it is rather immediate to predict the behavior of rational supervisor and subordinates in this interaction situation. It is a finite dynamic game with perfect information, with supervisor moving first, and then subordinates, acting simultaneously after observing the incentive. This game has a proper subgame starting from the information set of the subordinates. Therefore, there exists a set of subgame perfect Nash equilibria which equals the set of Nash equilibria that can be derived by backward induction. In fact, given any feasible incentives scheme $\left(b_{g}, b_{1}, b_{2}\right)$, the subordinates will

\footnotetext{
3 From the functional form of the production function it is immediate to observe that the two tasks are not additive; for a discussion the reader may refer to Spector (2003).

4 This notation is commonly used in the Game Theory literature, see for example Fudenberg and Tirole (1991).
} 
play a Nash equilibrium ${ }^{5}\left(u_{i}, l_{i}\right)$ of the subgame. Next, knowing the fact that subordinates will play a Nash equilibrium in the subgame, the supervisor will maximize her profit by choosing the optimal incentive scheme.

In the literature some factor or factors that keep agents from working infinitely hard are usually considered. For example, Wageman and Baker (1997) propose several mechanisms considering effort becoming increasingly either unproductive or unpleasant. In this paper, as is common, we assume that unpleasantness of the work increases with respect to the effort. Different cost functions may be considered and the functional form may reflect different underlying assumptions. While economics usually considers mainly rational agents, other approaches to work group dynamics take into account other aspects, such as norms and team commitment. Industrial and organizational psychology have proposed different theories of motivation to explain individual behavior in the organization (for a first survey, the reader may refer to Spector, 2003). Nevertheless, several other aspects underlie group and team behavior; the role of norms is well documented empirically (see Coch and French, 1948, and Roy, 1952, for example) and it is commonly assumed, for work groups, to dictate how much each person will produce (see Spector, 2003). A first cost function we consider is, as in Dal Forno and Merlone (2007), the following:

$$
c_{i}\left(u_{i}, l_{i}\right)=\left\{\begin{array}{r}
0 \text { if } u_{i}+l_{i} \leq \bar{c}_{i} \\
+\infty \text { if } u_{i}+l_{i}>\bar{c}_{i}
\end{array}\right.
$$

First observe that this cost function is non decreasing with respect to the aggregated effort. This kind of cost function assumes that each subordinate has a physical capacity $\bar{c}_{i}$ under which effort has zero cost, or, alternatively, that at some exertion level the effort becomes unpleasant enough to lead the individual to conclude that it is not worth working any harder independently of the reward.

In this case we assume that each individual knows his individual capacity and uses it without goldbricking. Finally, observe that this assumption can also be interpreted in terms of the self-efficacy theory (Bandura, 1982), assuming that both subordinates have high self-efficacy and are motivated to put in as much effort as they can.

$\overline{5}$ In the next sections we will examine also the equilibrium selection problem. 
As proved in Dal Forno and Merlone (2009b), when considering fully rational agents the optimal incentive scheme is

$$
\left\{\begin{array}{l}
b_{g}=\varepsilon>0 \\
b_{1}=0 \\
b_{2}=0
\end{array}\right.
$$

with effort allocation ${ }^{6}$ of subordinates

$$
\left(u_{i}, l_{i}\right)=\left(\frac{\alpha}{\alpha+\beta} \bar{c}_{i}, \frac{\beta}{\alpha+\beta} \bar{c}_{i}\right)
$$

for $i=1,2$.

\section{Modeling agents' behavior}

Since each agent can observe only the effort his colleague exerts with him, each subordinate's reaction can be formalized in terms of best reply function to the observed level effort. As it can be seen in Dal Forno and Merlone (2009b), in the one-shot game, among the infinitely many equilibria, there exists a focal one.

As discussed in Dal Forno and Merlone (2009b), for several reasons the optimal incentive scheme may represent a source of inequity perception from the point of view of the subordinates. For example, individuals with different capacity and, therefore, exerting different aggregate efforts, receive the same reward and, according to Adams (1965), may experience inequity. Furthermore, the differences each coworker finds when comparing his effort with the colleague can induce them to alter the effort allocation. In fact, according to Adams (1965) "Inequity exists for Person $(p)$ whenever he perceives that the ratio of his outcomes $(O)$ to inputs $(I)$ and the ratio of Other's $(o)$ outcomes to Other's input are unequal" (Adams, 1965, p.280). Formally, inequity exists whenever

$$
\frac{O_{p}}{I_{p}} \neq \frac{O_{o}}{I_{o}}
$$

${ }_{6}$ In Dal Forno and Merlone (2009b) it was proved that there exist infinitely many solutions among which one is rather natural and can be interpreted as focal in the sense of Schelling (1960). 
According to Adams, there are several possible consequences of inequity such as

(1) Person altering his inputs

(2) Person altering his outcomes

(3) Person distorting his inputs and outcomes cognitively

(4) Person leaving the field

(5) Person acting on other

(6) Person changing the objects of his comparison

Consistently with the literature on time and repeated inequity (see Cosier and Dalton, 1983), we assume that the subordinates, as the result of previous exposure to inequity, may no longer tolerate it.

Cosier and Dalton (1983) formalize the amount of tension experienced by Person due to the perceived inequity at discrete time $t$, as

$$
T(t)=\beta\left|\frac{O_{p}(t)}{I_{p}(t)}-\frac{O_{o}(t)}{I_{o}(t)}\right|
$$

where $\beta>0$ is a subjective proportionality factor which describes to what extent inequity causes tension in Person ${ }^{7}$. Cosier and Dalton correctly observe that a protocol, that does not account for past level of inequity, but time after time relies only on a sufficient current level, does not properly capture situations where agents overreact when face with what appears to be a relatively minor inequity. This situation is well described in section "The Straw that Broke the Camel's Back" in Cosier and Dalton (1983). For this reason, we assume, as they do, that the motivational strength ${ }^{8}$ aroused in Person to reduce tension depends on the current tension and previous motivational strength, and accumulates according to the formula

$$
M(t)=\alpha T(t)+\lambda M(t-1)
$$

where $\lambda>0$ is a discount factor, and $\alpha>0$ is a proportionality parameter that describes the degree to which tension motivates Person. This way, when the motivational strength $M(t)$ exceeds a subjective threshold $M^{*}$, subjects

$\overline{7}$ The formalization chosen in Cosier and Dalton (1983) reflects the fact that, according to Adams (1965), subjects react to inequity both when underpaid and overpaid. Obviously, how the individual acts in order to resolve the dissonance depends on the sign of inequity. But since in the following we consider effort allocation of intolerant individuals this aspect is irrelevant.

8 According to Spector (2003), motivation is defined as an internal state that induces a person to engage in particular behaviors. Motivational strength refers to the intensity of motivation. 
will act to reduce it. In the following, this is described by means of a subjective intolerance parameter defined as

$$
k(M(t), M(t-1), \ldots, M(0))=\left\{\begin{array}{l}
1 \quad \text { if } M(\tau)<M^{*}, \quad \tau=0,1, \ldots, t \\
\bar{k}>1 \quad \text { otherwise }
\end{array}\right.
$$

We assume that, not only the threshold $M^{*}$ is subjective, but also the value $\bar{k}$ of the intolerance parameter may be different among subordinates and may therefore concur in capturing their heterogeneity.

This can be interpreted as if the subordinates adopted a sort of grim strategy, which entails being tolerant to inequity until threshold is reached; once this occurs, intolerance remains at $\bar{k}>1$ for the rest of the interaction ${ }^{9}$. Moreover, the choice of considering grim strategies can also be interpreted in terms of conflict. While it is well known in the literature (see Steers and Black, 1994) that conflict is pervasive throughout organizations and that in some cases it may be good for organizations, on the contrary, in this case, as we will see in the next section, the conflict is dysfunctional. According to Steers and Black (1994), several conflict resolution techniques, which are often associated with an avoidance approach, are commonly found in organizations, even if they were proved to be ineffective (see Miles, 1980). We assume that the supervisor does not use any effective strategy to reduce the conflict. This can be either because she is unaware of the dysfunctional consequences of the conflict, or because she uses an avoidance approach, or, finally, because she lacks the resources to use a more effective strategy. As a consequence, we assume that, once the subjective threshold $M^{*}$ is exceeded, individual intolerance triggers and does not revert to tolerance.

In our analysis, we are interested in the dynamical process of the actions undertaken by subjects when the motivational strength exceeds the threshold. Subordinates, in order to reduce tension, reallocate their efforts on the two tasks, altering their inputs in Adams' formulation.

Dal Forno and Merlone (2007, 2009a) analyzed this interaction by means of human subject experiments. From their findings, it is possible to observe that almost all the subjects allocated their full capacity since the very beginning, and even those who did not, before the end of the experimental interaction used their full capacity too. Therefore, in the following we assume that subjects always allocate their full capacity. For these reasons, in order to describe the effort allocation dynamics, it is sufficient to consider only the level effort $l_{i}(t)$,

$\overline{9}$ For a first discussion on the grim strategy and other contingent strategies, the reader may refer to Dixit and Skeath (2004). 
since the effort exerted in the task with the supervisor is $u_{i}(t)=\bar{c}_{i}-l_{i}(t)$. The actions performed by the subordinates in order to reduce inequity can be modeled as follows. When $k_{i}=1$ the agent is tolerant to any colleague's deviation from the focal effort, i.e., whatever the level effort is, an agent with intolerance equal to 1 will always play the focal allocation. By contrast, as $k_{i}$ increases, the subordinate will decrease the lower effort as the colleague deviates from the expected focal allocation. In fact, when a subordinate observes the colleague exerting an effort which is lower than the focal one, he may think that his colleague is withholding the effort with him in order to work harder with the supervisor (impression management); his reaction will be to reduce the effort devoted in the common task and to increase his effort with the supervisor. Vice versa, when observing a colleague exerting an effort larger than the focal one, he will think that too little effort is put in the activity with the supervisor and will try to compensate for it. To summarize, basically an intolerant subordinate has two kinds of reaction depending on the fact the he observes the colleague exerting an effort which is lower or higher than the focal allocation. In the first case reducing his effort he reduces also the tension without considering the optimal production, in the second case he still tries to optimize the production. In order to derive a reaction function grounded on these two kinds of behavior let us assume for the moment that the focal allocation is unitary. In this case $l_{i}(t+1)=l_{-i}(t) e^{1-l_{-i}(t)}$ is a good approximation of the behavior we have just discussed since the two multiplicative factors respectively model the two behaviors and the focal allocation is a fixed point. In fact, factor $l_{-i}(t)$ models the subordinate's reaction when he perceives his colleague's effort to be lower than the focal one and factor $e^{1-l_{-i}(t)}$ models the reaction when colleague's effort is perceived to be larger than the focal one. Furthermore these kind of dynamics is quite common in the biological and ecological literature (see for instance May and Oster, 1976 and May, 1976). The second step is to introduce a parameter which models the intolerance of the subordinate; this can be obtained as follows

$$
l_{i}(t+1)=\left(l_{-i}(t)\right)^{k_{i}-1} e^{\left(k_{i}-1\right)\left(1-l_{-i}(t)\right)}
$$

Finally by a homothetic transformation we relax the assumption of a unitary focal allocation and obtain:

$$
l_{i}(t+1)=\frac{\beta \bar{c}_{i}}{\alpha+\beta}\left(\frac{(\alpha+\beta) l_{-i}(t)}{\beta \bar{c}_{i}}\right)^{k_{i}-1} e^{\left(k_{i}-1\right)\left(1-\frac{(\alpha+\beta) l_{-i}(t)}{\beta \bar{c}_{i}}\right)}
$$

As we can see, the reaction is a function of partner's effort $l_{-i}$ but also, via $k_{i}$, of the subordinate's intolerance to the accumulated tension to inequity.

Although rational economic agents are not supposed to have such a reaction, according to Baron and Kreps (1999), when considering employment relations 
the economic framework is silent on several aspects. In this sense, Gigerenzer and Selten (2002, p. 10) state that "Many decisions seem to be made on the basis of factors other than cognitive ones, that is, factors other than estimations of probabilities, gains, costs, and the like."

For instance, in our case the inequity described in Adams (1965) may elicit negative emotions, as it is evident from the example described by Cosier and Dalton (1983) on page 315.

Simon (1983, page 21) observes that

One thing an emotion can do for and to you is to distract you from your current focus of thought, and to call your attention to something else that presumably needs attention right now. Most of the time in our society we don't have to be out looking for food, but every so often we need to be reminded that food is necessary. So we possess some mechanisms that arouse periodically the feeling of hunger, to direct our attention to the need for food. A similar account can be given of other emotions.

The way we model the subordinates' reaction takes into account their reactions to inequity. In our case, as in Cosier and Dalton, tension elicits negative emotions which shape the subordinates' reaction as described above.

The functional form we consider is general enough to take into account both the case of subordinates who are tolerant to inequity, and those who were already put on the brink by their history of inequity (see Cosier and Dalton, 1983, p.315).

The reaction of subordinates to the effort their colleague exerts in the common task can be represented graphically as in Figure 1; in this case, we can observe that when $k_{i}=1$ the reaction functions become constant, i.e., the agent is tolerant to inequity.

In Section 2, we mentioned that the model allows for infinitely many equilibria. Any of these equilibria allows for the same production level which provides a comparative level for the work group production. In fact, from the effort allocation dynamics it is possible to compute the production and compare it to the optimal one.

The maximum production $\left(\frac{\alpha}{\alpha+\beta}\right)^{\alpha}\left(\frac{\beta}{\alpha+\beta}\right)^{\beta}\left(\bar{c}_{1}+\bar{c}_{2}\right)^{\alpha+\beta}$ is obtained when subordinates choose a focal allocation; furthermore, the production is null when aggregate effort -either with supervisor or between colleagues- is null. Therefore, it is rather natural to define the efficiency $\rho(t)$ as the ratio between actual 


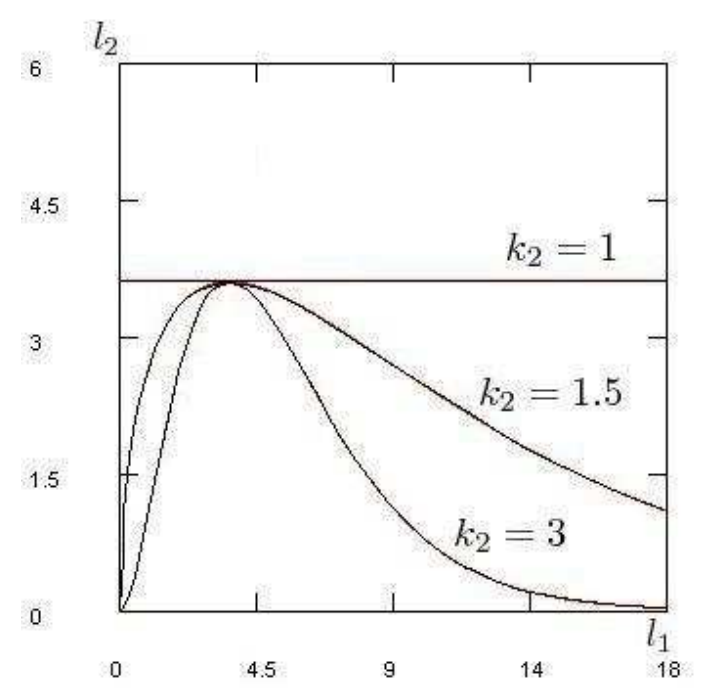

Fig. 1. Reaction function of subordinate 2 with: different values of the intolerance parameter $k_{2}=1, k_{2}=1.5, k_{2}=3$; elasticities $\alpha=0.7, \beta=0.3$; capacity $\bar{c}_{2}=12$.

and optimal production:

$$
\rho(t):=\frac{\left(u_{1}(t)+u_{2}(t)\right)^{\alpha}\left(l_{1}(t)+l_{2}(t)\right)^{\beta}}{\left(\frac{\alpha}{\alpha+\beta}\right)^{\alpha}\left(\frac{\beta}{\alpha+\beta}\right)^{\beta}\left(\bar{c}_{1}+\bar{c}_{2}\right)^{\alpha+\beta}}
$$

It varies in the range $[0,1]$ and, according to formula $(3)$, it is immediate to check that the efficiency is maximized when

$$
\left\{\begin{array}{l}
u_{1}(t)+u_{2}(t)=\frac{\alpha}{\alpha+\beta}\left(\bar{c}_{1}+\bar{c}_{2}\right) \\
l_{1}(t)+l_{2}(t)=\frac{\beta}{\alpha+\beta}\left(\bar{c}_{1}+\bar{c}_{2}\right)
\end{array}\right.
$$

Since we assume that agents use their full capacity, replacing $u_{i}=\bar{c}_{i}-l_{i}$ in (8), the efficiency can be written as

$$
\rho(t):=\frac{\left(\bar{c}_{1}-l_{1}(t)+\bar{c}_{2}-l_{2}(t)\right)^{\alpha}\left(l_{1}(t)+l_{2}(t)\right)^{\beta}}{\left(\frac{\alpha}{\alpha+\beta}\right)^{\alpha}\left(\frac{\beta}{\alpha+\beta}\right)^{\beta}\left(\bar{c}_{1}+\bar{c}_{2}\right)^{\alpha+\beta}}
$$

In the following section we examine the effort dynamics; considering also the efficiency index may shed some light on the consequences of these dynamics on the production level of the work group. 


\section{An analysis of the effort dynamics and the work group efficiency}

In the dynamics we consider, both subordinates' expectations are that colleague's allocation remains the same as in the current period and react accordingly ${ }^{10}$. The time evolution can be modeled by the iteration of a map $T:\left(l_{1}(t+1), l_{2}(t+1)\right) \rightarrow\left(r_{1}\left(l_{2}(t)\right), r_{2}\left(l_{1}(t)\right)\right)$ where $r_{1}, r_{2}$ are the reaction functions.

To analyze the effort dynamics it may be useful to consider three different cases, depending on the number of intolerant subordinates in the work group.

The analysis of the first case is rather straightforward. In fact, as long as $k_{1}=k_{2}=1$, the reaction functions are constant, and each player allocates his effort according to the focal equilibrium

$$
\left\{\begin{array}{l}
l_{1}(t+1)=\frac{\beta \bar{c}_{1}}{\alpha+\beta} \\
l_{2}(t+1)=\frac{\beta \bar{c}_{2}}{\alpha+\beta}
\end{array}\right.
$$

In this case the fixed point of the dynamics is the focal equilibrium (3). Nevertheless, when considering human subjects, evidence of such a dynamics is limited (see for instance Dal Forno and Merlone, 2007, 2009a). Furthermore, when the equity theory is considered, taking into account its dynamic nature as suggested in Cosier and Dalton (1983), differences in individual capacity may cause inequity. As a matter of fact, the different capacity of agents concurs in the accumulation of the tension due to inequity and, as a consequence, to an increasing of the motivational strength leading to a reallocation of efforts in order to resolve the tension.

When considering the interaction between one subordinate who is tolerant $\left(k_{i}=1\right)$, and one who is not $\left(k_{-i}>1\right)$, again there is a unique fixed point which is stable, since the eigenvalues of the Jacobian are both zero. In fact, in this case, assuming for the sake of simplicity that the tolerant subordinate is

$\overline{10}$ This kind of dynamic model -with naive expectations- was proposed by Cournot (1838) and has been studied by several authors. Recently, Bischi et al. (2000) have analyzes the properties of these duopoly games from the point of view of coexistence of attractors and structure of the basins. In the following we will examine how the theoretical properties of this kind of maps applies to the model of interaction we consider. 
the first one, the dynamics can be formalized as follows:

$$
\left\{\begin{array}{l}
l_{1}(t+1)=\frac{\beta \bar{c}_{1}}{\alpha+\beta} \\
l_{2}(t+1)=\frac{\beta \bar{c}_{2}}{\alpha+\beta}\left(\frac{(\alpha+\beta) l_{1}(t)}{\beta \bar{c}_{2}}\right)^{k_{2}-1} e^{\left(k_{2}-1\right)\left(1-\frac{(\alpha+\beta) l_{1}(t)}{\beta \bar{c}_{2}}\right)}
\end{array}\right.
$$

In this case, there are some consequences depending on the relative capacities of the two subordinates. When their capacity is identical, the fixed point corresponds to the focal equilibrium and the production is maximized. By contrast, in the other case, the intolerant agent will reallocate its effort, and when the tolerant subordinate is the one with lower capacity, the other subordinate may think that his colleague is withholding the effort with him in order to work harder with the supervisor. Vice versa, when the tolerant subordinate is the one with greater capacity, his colleague may think that the colleague is not working enough with the supervisor and will try to compensate for it. These considerations are illustrated in Figure 2.

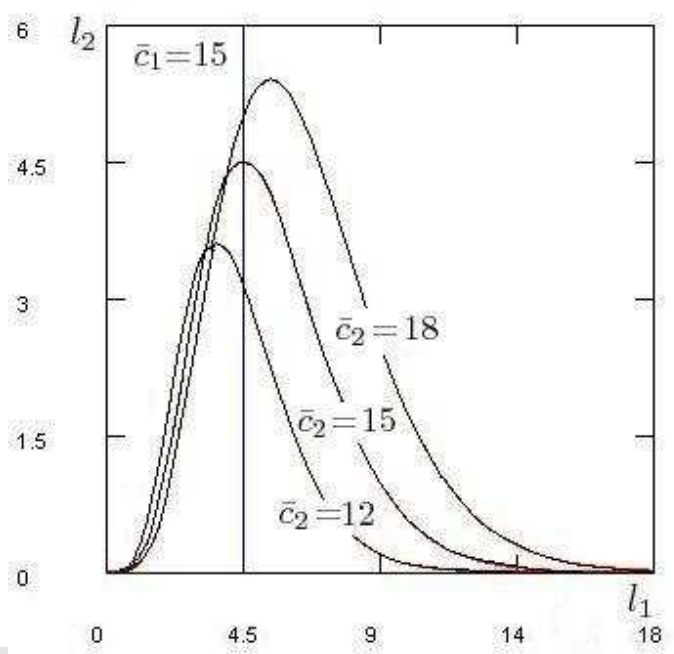

Fig. 2. Examples of the reaction function $r_{2}\left(l_{1}\right)$ with a unique tolerant subordinate $\left(\bar{c}_{1}=15\right)$ and different capacities for the intolerant agent $\left(\bar{c}_{2}=12\right.$, $\bar{c}_{2}=15$ and $\left.\bar{c}_{2}=18\right)$. The other parameter values are respectively: $\alpha=0.7, \beta=0.3, k_{1}=1, k_{2}=6$.

Furthermore, it is possible to observe that the efficiency is maximized when the capacities are identical, and the higher loss of efficiency occurs when the intolerant agent is the one with higher capacity. While we do not provide a formal proof of this result, we can observe it numerically, the intuition is immediate. In fact, since the higher capacity agent is the one who contributes more to the total production, the more he deviates from the focal effort allocation the lower is the production. 
Now we consider the case when, at a certain time $t$, both the subordinates have the motivational strength exceeding their individual threshold and, therefore, react to decrease the tension due to inequity. Here both values of the intolerance parameter are $k_{1}, k_{2}>1$. The effort allocation dynamics is given by

$$
\left\{\begin{array}{l}
l_{1}(t+1)=r_{1}\left(l_{2}(t)\right)=\frac{\beta \bar{c}_{1}}{\alpha+\beta}\left(\frac{(\alpha+\beta) l_{2}(t)}{\beta \bar{c}_{1}}\right)^{k_{1}-1} e^{\left(k_{1}-1\right)\left(1-\frac{(\alpha+\beta) l_{2}(t)}{\beta \bar{c}_{1}}\right)} \\
l_{2}(t+1)=r_{2}\left(l_{1}(t)\right)=\frac{\beta \bar{c}_{2}}{\alpha+\beta}\left(\frac{(\alpha+\beta) l_{1}(t)}{\beta \bar{c}_{2}}\right)^{k_{2}-1} e^{\left(k_{2}-1\right)\left(1-\frac{(\alpha+\beta) l_{1}(t)}{\beta \bar{c}_{2}}\right)}
\end{array}\right.
$$

and the analysis is more complex. While, in the previous cases, there exists only one fixed point, when $k_{1}, k_{2}>1$ the number of fixed points ranges from 1 to 3 , depending on the intolerance parameters value as it can be seen in Figure 3.

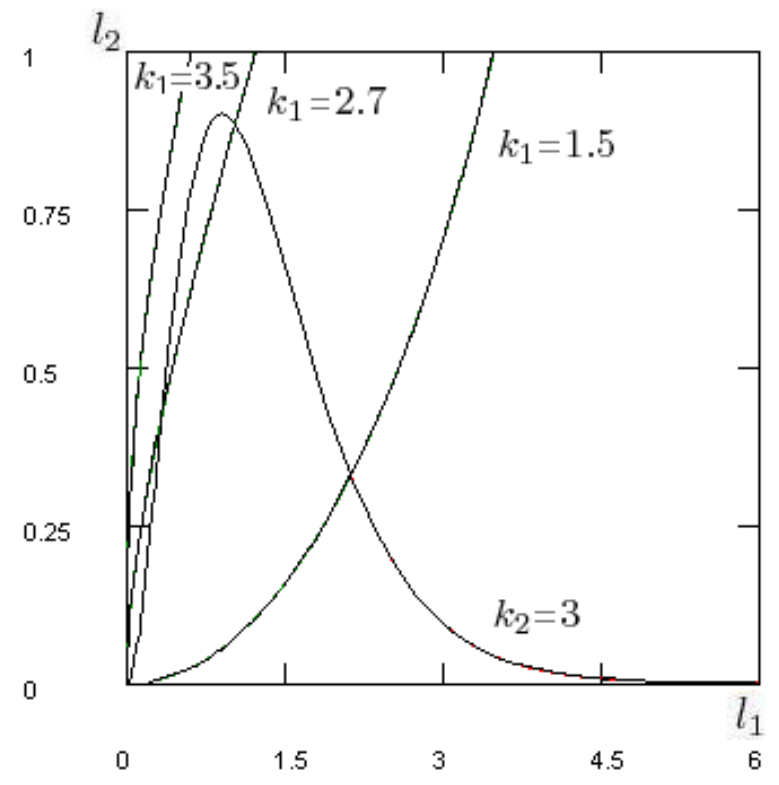

Fig. 3. Fixed points when both subordinates are not tolerant, with intolerance parameters $k_{1}=3.5, k_{1}=2.7$ and $k_{1}=1.5$ with $k_{2}=3$, and capacities $\bar{c}_{1}=18$ and $\bar{c}_{2}=3$.

Taking into account that $\alpha+\beta=1$, the Jacobian of (12) is

$$
\begin{aligned}
& J\left(l_{1}, l_{2}\right)= \\
& =\left(\begin{array}{ll}
0 & e^{\frac{\left(\beta \bar{c}_{1}-l_{2}\right)\left(k_{1}-1\right)}{\beta \bar{c}_{1}}}\left(\frac{l_{2}}{\beta \bar{c}_{1}}\right)^{k_{1}-2} \frac{\left(\beta \bar{c}_{1}-l_{2}\right)\left(k_{1}-1\right)}{\beta \bar{c}_{1}} \\
e^{\frac{\left(\beta \bar{c}_{2}-l_{1}\right)\left(k_{2}-1\right)}{\beta \bar{c}_{2}}}\left(\frac{l_{1}}{\beta \bar{c}_{2}}\right)^{k_{2}-2} \frac{\left(\beta \bar{c}_{2}-l_{1}\right)\left(k_{2}-1\right)}{\beta \bar{c}_{2}} & 0
\end{array}\right)
\end{aligned}
$$

It is immediate to observe that when the subordinates' intolerance is large 
enough, $(0,0)$ is a stable fixed point since the eigenvalues of the Jacobian are close to zero. On the contrary, when subordinates are tolerant $(0,0)$ is unstable. This can also be observed in Figure 1, when comparing the reaction curve to the first quadrant bisector. This corresponds to the case where both subordinates allocate their capacity exclusively with the supervisor and, therefore, the production is null; obviously, in terms of efficiency, this is the worst possible result.

It is not possible to analytically find the other intersections and, as a consequence, to compute the Jacobian in the steady states. Nevertheless the eigenvalues at $\left(l_{1}, l_{2}\right)$ are

$$
\left\{\begin{array}{l}
\lambda_{1}=-\sqrt{e^{\frac{\left(\beta \bar{c}_{1}-l_{2}\right)\left(k_{1}-2\right)}{\beta \bar{c}_{1}}+\frac{\left(\beta \bar{c}_{2}-l_{1}\right)\left(k_{2}-1\right)}{\beta \bar{c}_{2}}}\left(\frac{l_{2}}{\beta \bar{c}_{1}}\right)^{k_{1}-2} \frac{\left(\beta \bar{c}_{1}-l_{2}\right)\left(k_{1}-1\right)}{\beta \bar{c}_{1}}\left(\frac{l_{1}}{\beta \bar{c}_{2}}\right)^{k_{2}-2} \frac{\left(\beta \bar{c}_{2}-l_{1}\right)\left(k_{2}-1\right)}{\beta \bar{c}_{2}}} \\
\lambda_{2}=\sqrt{e^{\frac{\left(\beta \bar{c}_{1}-l_{2}\right)\left(k_{1}-2\right)}{\beta \bar{c}_{1}}+\frac{\left(\beta \bar{c}_{2}-l_{1}\right)\left(k_{2}-1\right)}{\beta \bar{c}_{2}}\left(\frac{l_{2}}{\beta \bar{c}_{1}}\right)^{k_{1}-2} \frac{\left(\beta \bar{c}_{1}-l_{2}\right)\left(k_{1}-1\right)}{\beta \bar{c}_{1}}\left(\frac{l_{1}}{\beta \bar{c}_{2}}\right)^{k_{2}-2} \frac{\left(\beta \bar{c}_{2}-l_{1}\right)\left(k_{2}-1\right)}{\beta \bar{c}_{2}}}}
\end{array}\right.
$$

From our analysis it is quite evident that, when both parameters $k_{i}$ are close to 1 , the dynamics is similar to the simple case analyzed in (10); in this case there are two fixed points: the origin which is stable and the one that is close to the efficient allocation, which, from the inspection of the eigenvalues, is stable too.

We can analyze the effects of subordinates' heterogeneity in terms of both capacity and intolerance contrasting the system behavior to the case of homogeneous subordinates. In fact when the subordinates are identical in terms of capacities and intolerance, i.e., $k_{1}=k_{2}=k$ and $\bar{c}_{1}=\bar{c}_{2}=\bar{c}$, then it is possible to prove that there are two equilibrium points, the null production equilibrium $(0,0)$ and the focal equilibrium

$$
l_{1}^{H}=l_{2}^{H}=\frac{\beta}{\alpha+\beta} \bar{c} .
$$

For some values of $k$, these symmetric equilibria are stable; there exists an unstable middle equilibrium $\left(l_{1}^{M}, l_{2}^{M}\right)$ between these two equilibria as in Figure 4. In this figure we show the basins of the two fixed points and of the period-2 cycle, together with a trajectory for each case.

When subordinates are heterogeneous in terms of capacity the situation is different as illustrated in Figure 5 . In this case capacities are respectively $c_{1}=$ $18, c_{2}=6$. The result is counterintuitive since, even if one of the subordinates's capacity increases, the basin of the focal non null equilibrium shrinks, and, paradoxically, even if the intolerance remains the same it is more likely that with the higher capacity subordinate the interaction switches to a retaliation 
cycle or, even worse to null production. Finally, it is important to observe that, even if the intolerance value is the same, when the capacity is slightly different the values of $l_{1}^{H}$ and $l_{2}^{H}$ are no longer focal and can only be obtained numerically ${ }^{11}$.

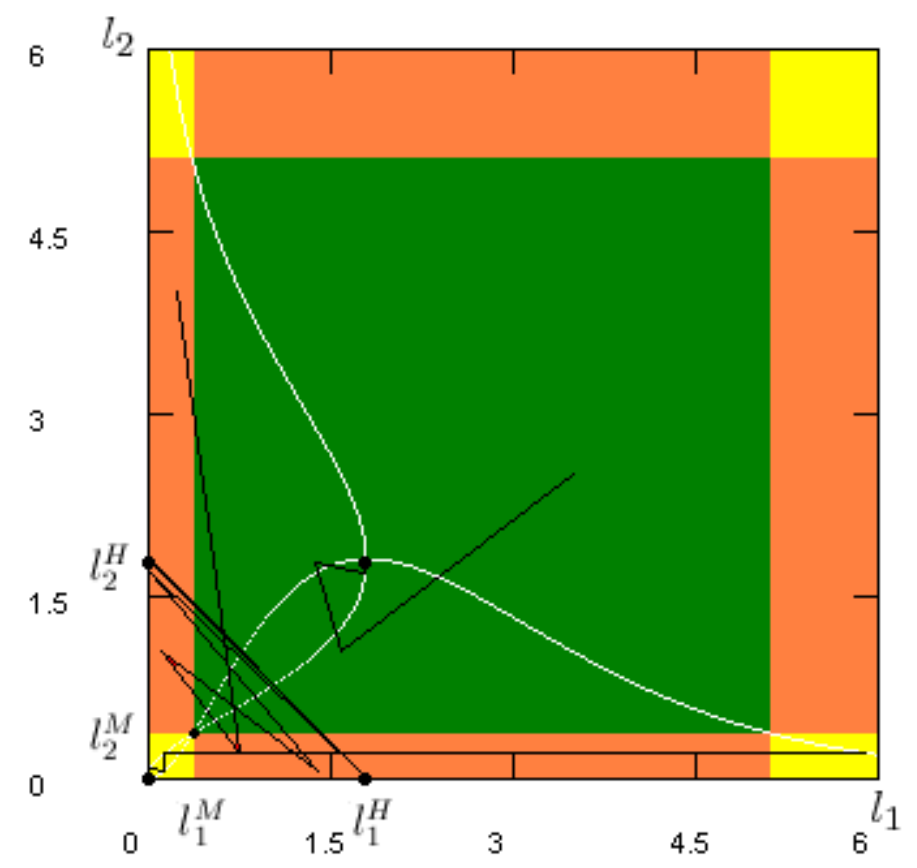

Fig. 4. Phase plane $\left(l_{1}, l_{2}\right)$ with periodic attractors and their basins for $k_{1}=k_{2}=3$, with capacity values $\bar{c}_{1}=6, \bar{c}_{2}=6$. The initial conditions of the trajectories are respectively $l_{1}(0)=3.5, l_{2}(0)=2.5$ (fixed point), $l_{1}(0)=0.25, l_{2}(0)=4.0(2-$ period cycle), and $l_{1}(0)=5.9, l_{2}(0)=0.2$ (origin) .

Considering subordinates' heterogeneity also in terms of different values of tolerance, ie., we no longer expect them to have the same value for $k_{i}$, we can observe quite different dynamics from the previous one. Figure 6 shows the bifurcation diagrams of the subordinates effort, as the intolerance of the first subordinate ranges from 1.0 to 2.2 , when confronting a lower capacity agent who is rather intolerant $\left(k_{2}=7.5\right)$. As it is well known for nonlinear maps, we have the coexistence of several attracting sets and their respective basin of attraction. The dynamic process becomes path dependent and a problem of equilibrium selection arises. In this case we can see that as the subordinate intolerance grows, a cascade of flip bifurcations leads to chaos. This proves that, when one subordinate is intolerant and the other is less, it is possible to converge to a cycle with periodicity higher than two up to chaos. Finally, as $k_{1}$ increases further, both subordinates end up exerting all the effort with the supervisor; in this case the work group efficiency is null ${ }^{12}$. The whole diagram

$\overline{11}$ We are grateful to an anonymous referee for this observation.

12 It is possible to observe that this transition occurs since the basin of the null 
is not symmetric since at the beginning the effort is not null, while it may be so for $k_{1}$ large enough. In fact we see that when both the subordinates are very intolerant the unique equilibrium is $l_{1}=l_{2}=0$.

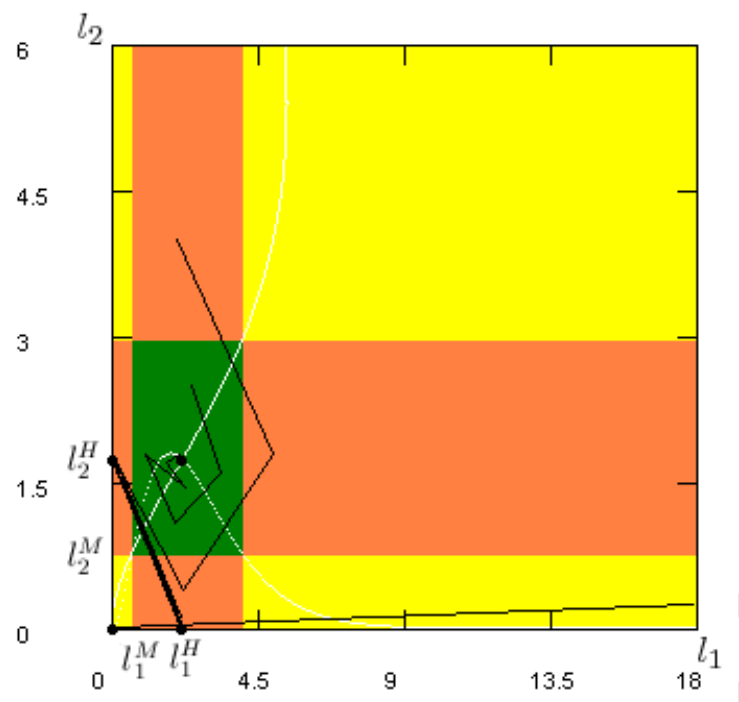

Fig. 5. Phase plane $\left(l_{1}, l_{2}\right)$ with periodic attractors and their basins for $k_{1}=k_{2}=3$, with parameters values $\bar{c}_{1}=18, \bar{c}_{2}=6$. The initial conditions of the trajectories are respectively $l_{1}(0)=2.5, l_{2}(0)=2.5$ (fixed point), $l_{1}(0)=2.0, l_{2}(0)=4.0(2$-period cycle), and $l_{1}(0)=17.9, l_{2}(0)=0.25$ (origin).
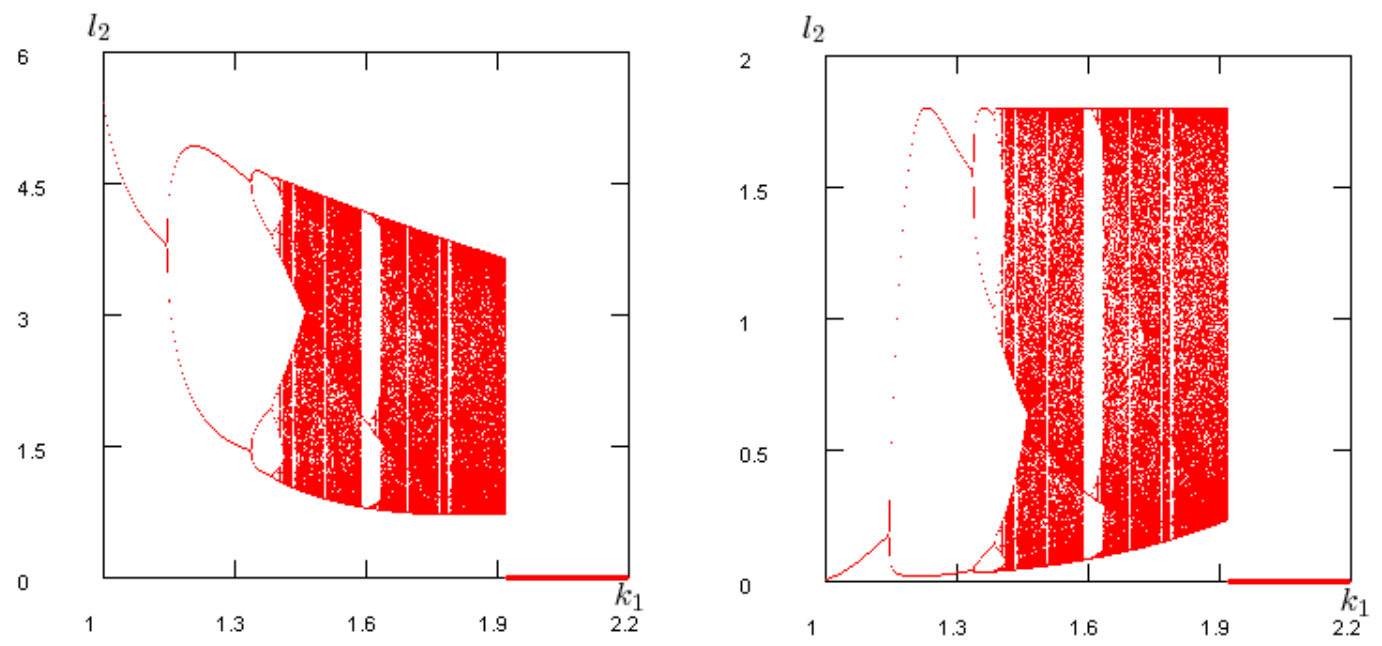

Fig. 6. Bifurcation diagrams of subordinates 1's effort (left) and 2's effort (right) as $k_{1} \in[1.0,2.2]$, with parameters values $k_{2}=7.5, \bar{c}_{1}=18, \bar{c}_{2}=6$, and initial condition $l_{1}(0)=l_{2}(0)=.1$.

We assume now that the low capacity agent is rather intolerant $\left(k_{2}=7.5\right)$.

equilibrium enlarges; the figure illustrating this phenomenon are omitted for the sake of brevity. 
It can be observed that, as parameter $k_{1}$ increases from 1.39 to 1.7 , a periodic cycle becomes chaotic as illustrated in Figure 7. Black dots indicate the sequence of periodical points visited at each iteration, while the joining lines are depicted for illustrative purpose only. From the organization point of view, we observe that, as long as the first agent intolerance is limited $\left(k_{1}=1.39\right)$, the interaction follows a pattern which repeats -a cycle with finite period- and therefore is predictable. Vice versa, when both the subordinates are rather intolerant $\left(k_{1}=1.7\right)$, the interaction never repeats -a chaotic trajectory.
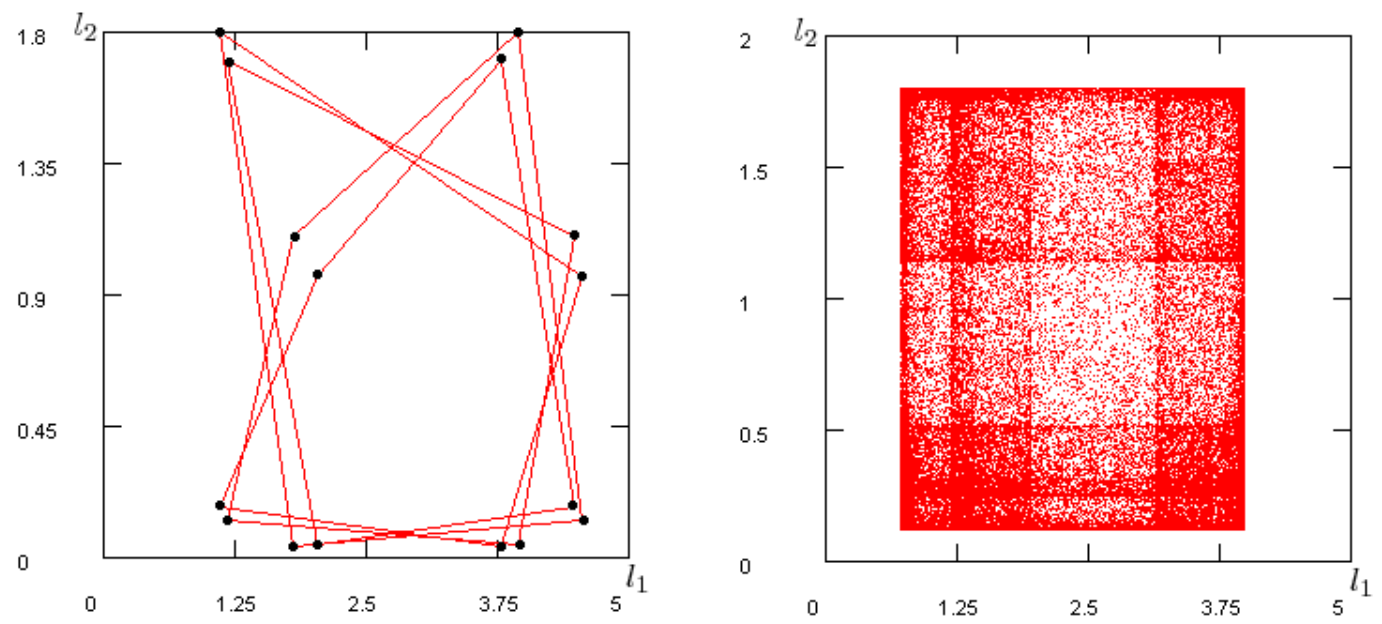

Fig. 7. Different attractors: 16 -period with $k_{1}=1.39$ (left) and chaotic with $k_{1}=1.7$ (right). The other parameters values are $\bar{c}_{1}=18, \bar{c}_{2}=6, k_{2}=7.5$, initial condition $l_{1}(0)=1.12, l_{2}(0)=.17$.

In Figure 8 we can see some of the coexisting finite period cycles. Again, as in Figure 7, black dots indicate the sequence of periodical points visited at each iteration. In particular, the picture at bottom right shows the retaliation actions. The respective basins of attraction are reported on top left. In this case there are five 16-period stable cycles. The computation of the periodic points, as well as the structure of the basins, has been studied in Bischi et al. (2000). Following their terminology, we can say that four of them are homogeneous cycles deriving from a stable 8-period cycle of the map $F(x)=r_{1}\left(r_{2}(x)\right)$, one of which -bottom left in the figure- is a Markov-Perfect-Equilibrium (MPE), the last one is a mixed 16-period cycle which derives from the fixed point in the origin. Several authors have discussed MPE in oligopoly dynamics, see for example Maskin and Tirole (1988a, 1988b), and Bischi et al. (2000). In our case the MPE is interesting in terms of group dynamics. In fact, this equilibrium represents a situation in which at any discrete time only one of the subordinates changes his effort allocation. This means that each subordinate at every other turn believes that his previous allocation is optimal. In this case, the allocation dynamics is midway between a constant allocation (a fixed point) and a situation in which both subordinates adjust their allocation at each period. Finally, also the mixed 16-period cycle deriving from the fixed 
point in the origin is interesting in terms of group dynamics as, in this case in turn, one of the subordinates stops cooperating with his colleague. This sort of sequence retaliation may be interpreted in terms of intergroup conflict. The coexistence of these two different dynamics well illustrates why the term "complexity" is so popular when trying to explain group dynamics (for a reference of complexity in social psychology see Arrow et al., 2000 and Nowak and Vallacher, 1998).
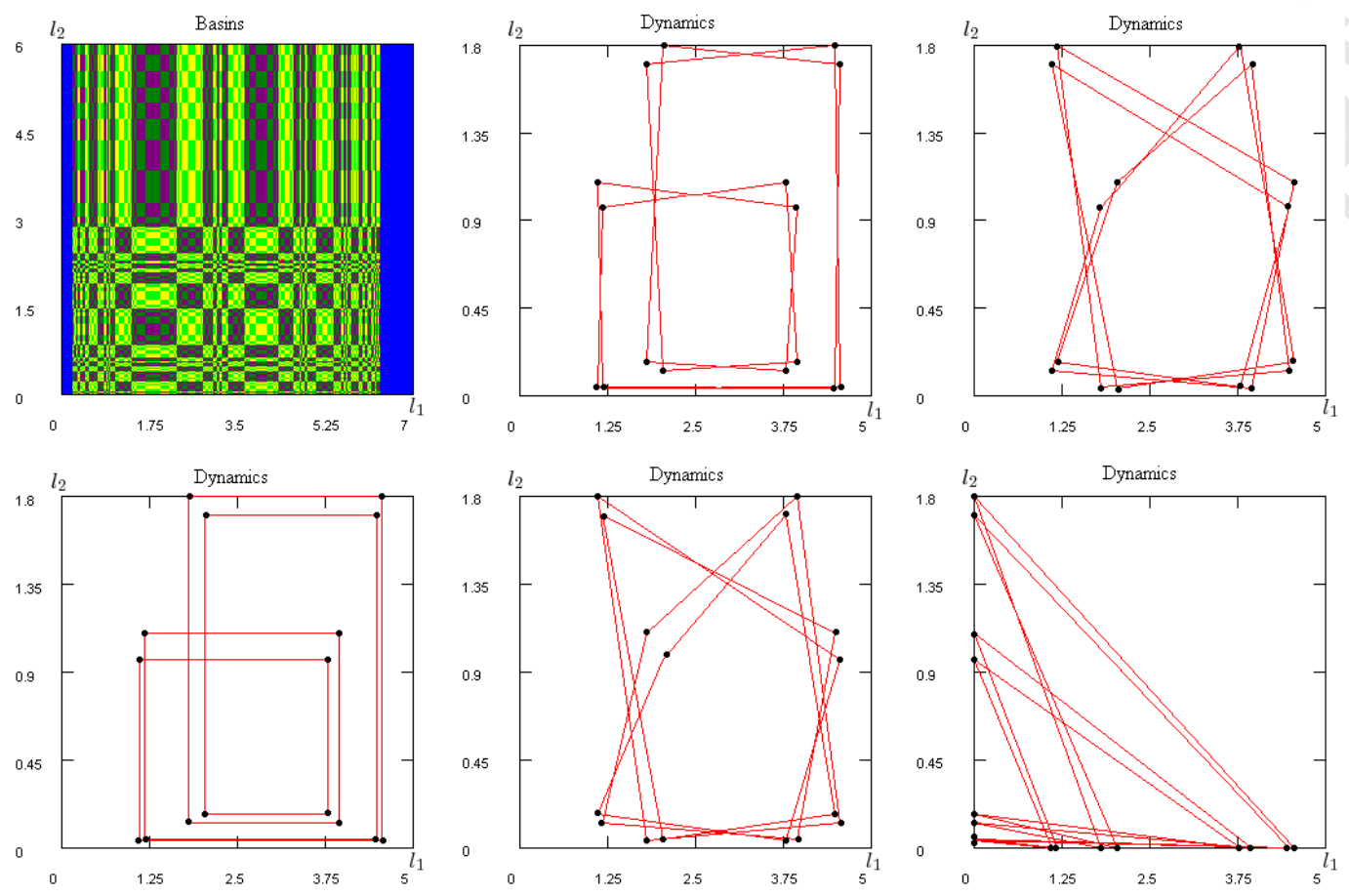

Fig. 8. Basins of attraction and respective periodic cycles with $\bar{c}_{1}=18, \bar{c}_{2}=6$, $k_{1}=1.39, k_{2}=7.5$. On the bottom left a MPE is illustrated, while a retaliation cycle is at the bottom right.

As we mentioned above, Bischi et al. (2000) examined MPE oligopoly dynamics, and, more generally, provided several properties holding for maps with the form $T:(x, y) \rightarrow\left(r_{1}(y), r_{2}(x)\right)$. Since the map we are considering in this paper has the same form, all these properties apply in our case as well. For example, it is known (Proposition 4 in Bischi et al., 2000) that, for any periodic point $P$ of the map $T$ with period $n \geq 2$, the parallel lines to the axes issuing from $P$ are trapping sets for the map $T^{n}$, that is, we have a recurrent allocation. This means that, at least one subordinate will repeat the same allocation in the future. Therefore, the supervisor, in order to modify dysfunctional and inefficient dynamics, can exploit recurrent allocations to promptly adopt interventions with the work group.

If we repeat such a numerical analysis, we observe other interesting results. For instance, the diagram of bifurcation -which is similar to Figure 6 and therefore, for the sake of brevity, is not reported here- when the capacity of 
the high capacity agent is made to vary, presents a sequence of flip bifurcations. But when the parameter is the capacity of the low capacity subordinate, the diagram of bifurcation exhibits, firstly, a chaotic behavior which, later on, collapses into a reverse flip bifurcation, ending with a unique fixed point.

Since multistability occurs, it is interesting to characterize the set of initial conditions leading to each coexisting attractor, i.e., to determine the basins of the attractors. We can observe that the basin of attraction of the origin (which corresponds to the worst case for the work group, because both the subordinates are exerting an effort with the supervisor only, and therefore the production is null) enlarges, and it is no more connected, when $k_{1}$ increases. Figure 9 illustrates this situation. The basin of attraction of the origin, when $k_{1}=1.39$ is a small horizontal rectangular region which includes $O=(0,0)$. But when the level of intolerance of the higher capacity agent increases to $k_{1}=1.503$, even starting close to $A(5.1607,3.4321)$ it is possible to converge to the origin where the production is null. This may explain how, depending only on some not directly observable parameters such as, in this case, the intolerance, from apparently identical initial conditions work group dynamics may be quite different.
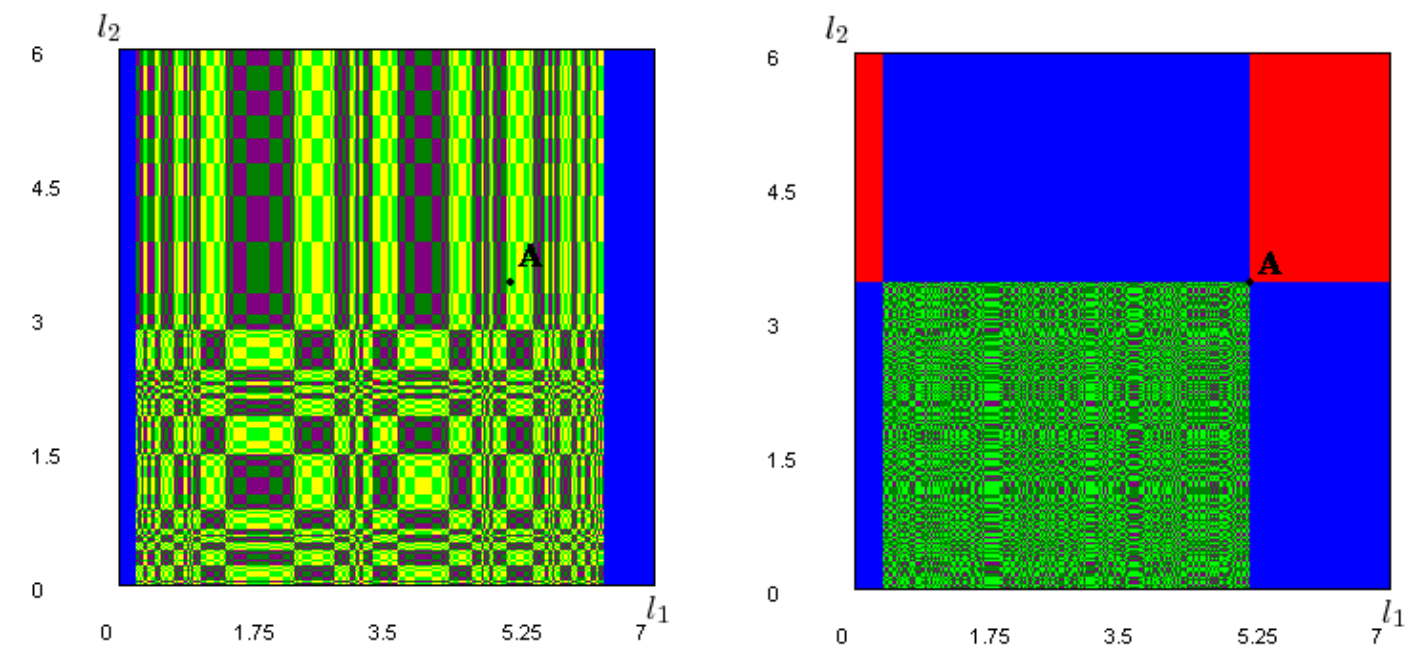

Fig. 9. Basins of attraction with $\bar{c}_{1}=18, \bar{c}_{2}=6, k_{1}=1.39, k_{2}=7.5$ (left) and $\bar{c}_{1}=18, \bar{c}_{2}=6, k_{1}=1.503, k_{2}=7.5$ (right). The black dot indicates initial condition $A(5.1607,3.4321)$.

\section{Conclusion}

In this paper we considered the effects of inequity in a work group. In particular, following Cosier and Dalton (1983), we assume that, as a result of the history of inequity individuals have been exposed to, the subordinates allocate their efforts in order to reduce the inequity. The analysis of the different cases 
gives some results in terms of efficiency and effort dynamics. We summarize our results comparing the case in which subordinates' capacities are the same to the case where they differ.

From our results it follows that, in the first case, i.e., when the subordinates have the same capacity, if at least one of them does not use effort reallocation to reduce the inequity, then the work group maximizes its production. On the other hand, when both subordinates are intolerant we can have coexistence of attractors: the focal solution coexists with retaliation 2-period cycles.

The situation is quite different when subordinates' capacity is different. In fact, while in this case when both the subordinates are tolerant the focal solution remains the only equilibrium, when one tolerant subordinate faces an intolerant colleague the work group loses efficiency, even if retaliation cycles do not trigger. Finally, when both subordinates are intolerant, several different cycles may coexist, even with retaliation, and finally, for sufficiently large values of intolerance, chaotic cycles occur. For even larger values the basin of the null production equilibrium expands and becomes not connected. The interpretation of this latter case is interesting in terms of work group dynamics as it means that the same initial condition may, depending on the subordinates' intolerance, lead either to a cycle or to the null production.

These latter dynamics may be considered as examples in which the primary task (Miller and Rice, 1967) of the group shifts from production to conflict between subordinates. In these cases some timely interventions may be necessary in order to make members aware of the normative primary task (Roberts, 1994).

In further research it would be interesting to assume that the intolerance parameters may vary over time, including cases in which subordinates' motivation to reduce inequity may become lower, for example when inequity decreases. This would lead to more complex patterns of behavior where it is possible to evaluate also the effect of other techniques for reducing conflict.

\section{References}

Adams, J.S., 1965. Inequity in social exchange. In: Berkowitz, L. (Ed.). Advances in Experimental Social Psychology. New York: Academic Press, 276299.

Arrow, H., McGrath, J.E., Berdahl, J.L., 2000. Small Groups as Complex Systems: Formation, Coordination, Development, and Adaptation. , Beverly Hills: Sage Publications.

Bandura, A., 1982. Self-efficacy mechanism in human agency. American Psychologist 37, 122-147. 
Baron, J.N., Kreps, D.M., 1999. Strategic Human Resources: Framework for General Managers. New York: John Wiley \& Sons.

Bischi, G.I., Mammana, C., Gardini, L., 2000. Multistability and cyclic attractors in duopoly games. Chaos, Solitons and Fractals 11, 543-564.

Campbell, J.P., Pritchard, R.D., 1976. Motivation theory in industrial and organizational psychology. In: Dunnette, M.D. (Ed.). Handbook of Industrial and Organizational Psychology. Chicago: Rand McNally, 63-130.

Coch, L., French, J.R.P. Jr., 1948. Overcoming resistance to change. Human Relations 1, 512-532.

Cosier, R.A., Dalton, D.R., 1983. Self-efficacy mechanism in human agency. Equity theory and time: a reformulation. Academy of Management Review 8, 311-319.

Cournot, A., 1838. Recherches sur les Principes Mathematiques de la Theorie des Richesses. Hachette, Paris. (English translation, 1960). Researches into the Mathematical Principles of the Theory of Wealth. New York: Kelly.

Cropanzano, R., Schminke, M., 1995. Using social justice to build effective work groups. In: Turner, M. (Ed.). Groups at Work: Advances in Theory and Research. Hillsdale: Erlbaum, 143-171.

Dal Forno, A., Merlone, U., 2007. Incentives in supervised teams: an experimental and computational approach. Journal of Social Complexity 3, 37-52.

Dal Forno, A., Merlone, U., 2009a. Individual incentives in supervised work groups. From human subject experiments to agent based simulation. International Journal of Internet and Enterprise Management 6, 4-22.

Dal Forno, A., Merlone, U., 2009b. Individual-based versus Group-based incentives in supervised work groups. The role of individual motivation. Submitted manuscript.

Dixit, A., Skeath, S., 2004. Games of Strategy (2nd Edition). New York: W. W. Norton \& Company.

Fudenberg, D., Tirole, J., 1991. Game Theory. Cambridge: MIT Press.

Gigerenzer, G., Selten, R., 2002. Rethinking rationality. In: Gigerenzer, G., Selten, R. (Eds.). Bounded Rationality: The Adaptive Toolbox. Cambridge: The MIT Press, 1-12.

Maskin E., Tirole, J., 1988a. A theory of dynamic oligopoly I: overview and quantity competition with large fixed costs. Econometrica 56, 549-570. 
Maskin E., Tirole, J., 1988b. A theory of dynamic oligopoly II: price competition. Econometrica 56, 571-600.

Maslow, A.H., 1970. Motivation and Personality. New York: Harper \& Row.

May, R.M., 1976. Simple mathematical models with very complicated dynamics. Nature 261, 459-467.

May, R.M., Oster, G.F., 1976. Bifurcations and dynamic complexity in simple ecological models. The American Naturalist 110, 573-599.

Miles, R., 1980. Macro Organizational Behavior. Glenview: Scott, Foresman.

Miller, E.J., Rice, A.K., 1967. Systems of Organization. London: Tavistock Publications.

Mowday, R.T., 1979. Equity theory predictions of behavior in organizations. In: Steers, R.M., Porter, L.W. (Eds.). Motivation and Work Behavior. New York: McGraw-Hill, 124-146.

Nowak, A., Vallacher, R.R., 1998. Dynamical Social Psychology. New York: The Guildford Press.

Roberts, V.Z., 1994. The organization of work. In: Obholzer, A., Roberts, V.Z. (Eds.). The Unconscious at Work. London: Routledge, 28-38.

Roy, D., 1952. Quota restriction and goldbricking in a machine shop. The American Journal of Sociology 57, 427-442.

Schelling, T.C., 1960. The Strategy of Conflict. Cambridge: Harvard University Press.

Simon, H.A., 1983. Reason in Human Affairs. Stanford: Stanford University Press.

Spector, P.E., 2003. Industrial and Organizational Psychology (3rd Edition). New York: John Wiley \& Sons.

Steers, R.M., Black, J.S., 1994. Organizational Behavior (5th Edition). New York: Harper Collins.

Wageman R., Baker, G., 1997. Incentives and cooperation: the joint effects of task and reward interdependence on group performance. Journal of Organizational Behavior 18, 139-158. 